\title{
Optimisation of chemotherapy in the era of immunotherapy
}

\author{
René Boosman ${ }^{1}$ and Jacobus (Sjaak) Adrianus Burgers ${ }^{2}$
}

Affiliations: ${ }^{1}$ Dept of Pharmacy and Pharmacology, Netherlands Cancer Institute, Amsterdam, The Netherlands. ${ }^{2}$ Thoracic Oncology, Netherlands Cancer Institute, Amsterdam, The Netherlands.

Correspondence: Jacobus (Sjaak) Adrianus Burgers, Thoracic Oncology, Netherlands Cancer Institute, Plesmanlaan 121, Amsterdam, The Netherlands. E-mail: s.burgersanki.nl

@ERSpublications

Even in the era of immuno- and personalised therapy, optimal use of old-fashioned chemotherapy is of utmost importance, also for patients with renal insufficiency or declining renal function http://ow.ly/rdlH30m4V1N

Cite this article as: Boosman R, Burgers JA. Optimisation of chemotherapy in the era of immunotherapy. Eur Respir J 2018; 52: 1801698 [https://doi.org/10.1183/13993003.01698-2018].

Even in the era of immunotherapy and personalised medicine, chemotherapy remains a cornerstone in the treatment of nonsmall cell lung cancer (NSCLC) [1]. Chemotherapy improves the length and quality of life of patients with metastatic disease for tumours both with and without oncogenic drivers. Chemotherapy in combination with checkpoint inhibitors is about to become the most effective first-line therapy for NSCLC [2]. Also in the adjuvant setting, i.e. adding chemotherapy after surgery, chemotherapy improves survival with an absolute increase of $4 \%$ at 5 years [3].

The optimal use of chemotherapy is an important factor in optimising the prognosis for patients. The fear of, or actual occurrence of, side-effects might interfere with its optimal use. A common complication of chemotherapy is the development of renal failure. The decline in renal function can occur as a direct toxic effect of the chemotherapeutic agent, but also patient-related and other drug-related factors play pivotal roles [4]. Nephrotoxicity often is a reason of treatment discontinuation or dose reduction resulting in a suboptimal treatment schedule [5]. The fact that about $60 \%$ of the people with cancer have underlying compromised renal function stresses the importance of this topic [6].

In this issue of the European Respiratory Journal, VISSER et al. [7] describe the impact of pemetrexed on the renal function of patients with NSCLC. Pemetrexed is a therapeutic option for many patients. It is currently approved for treatment of nonsquamous NSCLC and mesothelioma. The approval for nonsquamous NSCLC involves first-line therapy in combination with cisplatin, and more recently in combination with carboplatin and pembrolizumab, as continuation and switch maintenance treatment and second-line therapy. In mesothelioma, the pemetrexed-cisplatin combination is the only approved regimen [8].

In the currently approved dose of $500 \mathrm{mg} \cdot \mathrm{m}^{-2}$, pemetrexed pharmacokinetics are linear. It is eliminated via the kidneys, with $70 \%-90 \%$ of the administered drug recovered in the urine within $24 \mathrm{~h}$ [9], it shows a biphasic elimination, and pemetrexed clearance linearly correlates with creatinine clearance [10, 11]. Systemic exposure is importantly correlated with toxicity and efficacy [12, 13], with a higher exposure leading to a higher incidence of dose-limiting haematological toxicity [10]. Renal function and dose of pemetrexed are the sole determinants for total systemic exposure [10, 14, 15].

Although nephrotoxicity is not amongst the list of dose-limiting toxicities of pemetrexed [16], it is commonly encountered. Pemetrexed enters the proximal tubule cells both via the basolateral and the 
apical side. Inside the cells pemetrexed is polyglutamylated, which impairs transport of pemetrexed out of the cell and results in raised intracellular concentrations. The inhibition of enzymes involved in the folate pathway by pemetrexed, impairing DNA and RNA synthesis of the tubule cells, further adds to the nephrotoxic effect of pemetrexed [4].

Presently, VisSER et al. [7] showed in their prospective study, which was performed in a standard hospital setting, that patients with an estimated glomerular filtration rate (eGFR) of $<90 \mathrm{~mL} \cdot \mathrm{min}^{-1}$ prior to the treatment with pemetrexed were at increased risk to develop acute kidney disease. The authors were able to confirm this observation in an independent retrospective cohort of NSCLC patients. Both cohorts also showed that a decrease in renal function during first-line pemetrexed-platinum treatment predicted for the development of renal disease during maintenance pemetrexed. During the maintenance treatment, almost $30 \%$ of the patients developed a decline in renal function, of whom $60 \%$ had to stop chemotherapy. The authors also noticed a statistically nonsignificant relationship between the cumulative dose of pemetrexed and nephrotoxicity.

It is obvious that renal toxicity has more impact on patients with a pre-existing impaired renal function. Whether this patient population also were more likely to develop significant nephrotoxicity has already been suggested in other studies. The paper by VISSER et al. [7], however, is the first to show the predictive properties of baseline reduced eGFR and reduced renal function during therapy for a (further) reduction in renal function due to pemetrexed. This is in line with a similar French study which had shown that renal toxicity was the main reason for interruption of treatment with pemetrexed and bevacizumab [17].

The study by VISSER et al. [7] has limitations, related to its partially retrospective design, the fact that the effect of pemetrexed was studied when given in combination with the nephrotoxic agents carboplatin and cisplatin, the lack of data on concomitant medication, and the relatively small number of patients. Nonetheless, the study paves the way for new strategies and research ideas and once again underlines the importance of optimal treatment for all patients, including those with a renal impairment.

A way to improve care for this friable population might be a change of our standard dosing practice. The current, standard practice of pemetrexed dosing on body surface area, by which the renal function is not taken into account, confronts the clinicians with two major problems: 1) a potentially effective treatment is withheld from patients with an eGFR $<45 \mathrm{~mL} \cdot \mathrm{min}^{-1}$ [11]; and 2) deterioration of renal function as a result of pemetrexed treatment, leading to adverse effects and cessation of the treatment $[11,18]$, might prevent optimal anti-tumour therapy, as is also shown by VISSER et al. [7]. This applies both to those patients with normal renal function and to those patients with a diminished renal function at the start of therapy $[4,9]$.

New studies on individualised pemetrexed dosing in patients with NSCLC and mesothelioma based on their renal function are, therefore, being eagerly awaited, also in the new immunotherapy era, in which triple combinations of chemotherapy and immunotherapy are about to become standard of NSCLC care.

Conflict of interest: R. Boosman has nothing to disclose. J.A. Burgers reports advisory board work for Roche, Boehringer Ingelheim and AstraZeneca, outside the submitted work.

\section{References}

1 Hellman MD, Li BT, Chaft JE, et al. Chemotherapy remains an essential element of personalized care for persons with lung cancers. Ann Oncol 2016; 10: 1829-1835.

2 Gandhi L, Rodríquez-Abreu D, Gadgeel S, et al. Pembrolizumab plus chemotherapy in metastatic non-small-cell lung cancer. N Engl J Med 2018; 378: 2078-2092.

3 Burdett S, Pignon JP, Tierney J, et al. Adjuvant chemotherapy for resected early-stage non-small cell lung cancer. Cochrane Database Syst Rev 2015; 2: CD011430.

4 Perazella MA. Onco-nephrology: renal toxicities of chemotherapeutic agents. Clin J Am Soc Nephrol 2012; 7: 1713-1721.

5 Sbitti Y, Chahdi H, Slimani K, et al. Renal damage induced by pemetrexed causing drug discontinuation: a case report and review of the literature. J Med Case Rep 2017; 11: 182.

6 Sahni V, Choudhury D, Ahmed Z. Chemotherapy-associated renal dysfunction. Nat Rev Nephrol 2009; 5: 450-462.

7 Visser S, Huisbrink J, van ' $t$ Veer NE, et al. Renal impairment during pemetrexed maintenance in patients with advanced nonsmall cell lung cancer: a cohort study. Eur Respir J 2018; 52: 1800884.

8 Vogelzang NJ, Rusthoven JJ, Symanowski J, et al. Phase III study of pemetrexed in combination with cisplatin versus cisplatin alone in patients with malignant pleural mesothelioma. J Clin Oncol 2003; 21: 2636-2644.

9 Physicians' Desk Reference. Alimta, Pemetrexed for Injection. Thomson PDR, Montvale, $2006 ;$ p. 1722.

10 Latz JE, Chaudhary A, Ghosh A, et al. Population pharmacokinetic analysis of ten phase II clinical trials of pemetrexed in cancer patients. Cancer Chemother Pharmacol 2006; 57: 401-411.

11 European Medicines Agency (EMA). ALIMTA EPAR - Product Information. 2017.

12 Ikesue $\mathrm{H}$, Watanabe $\mathrm{H}$, Hirano $\mathrm{M}$, et al. Risk factors for predicting severe neutropenia induced by pemetrexed plus carboplatin therapy in patients with advanced non-small cell lung cancer. Biol Pharm Bull 2015; 38: 1192-1198. 
13 Latz JE, Rusthoven JJ, Karlsson MO, et al. Clinical application of a semimechanistic-physiologic population PK/ PD model for neutropenia following pemetrexed therapy. Cancer Chemother Pharmacol 2006; 57: 427-435.

14 Ouellet D, Periclou AP, Johnson RD, et al. Population pharmacokinetics of pemetrexed disodium (ALIMTA) in patients with cancer. Cancer Chemother Pharmacol 2000; 46: 227-234.

15 Chen CY, Lin JW, Huang JW, et al. Estimated creatinine clearance rate is associated with the treatment effectiveness and toxicity of pemetrexed as continuation maintenance therapy for advanced nonsquamous non-small-cell lung cancer. Clin Lung Cancer 2015; 16: e131-e140.

16 Takimoto CH, Hammond-Thelin LA, Latz JE, et al. Phase I and pharmacokinetic study of pemetrexed with high-dose folic acid supplementation or multivitamin supplementation in patients with locally advanced or metastatic cancer. Clin Cancer Res 2007; 13: 2675-2683.

17 Sassier M, Dugué AE, Clarisse B, et al. Renal insufficiency is the leading cause of double maintenance (bevacizumab and pemetrexed) discontinuation for toxicity to advanced non-small cell lung cancer in real world setting. Lung Cancer 2015; 89: 161-166.

18 Middleton G, Gridelli C, De Marinis F, et al. Evaluation of changes in renal function in PARAMOUNT: a phase III study of maintenance pemetrexed plus best supportive care versus placebo plus best supportive care after induction treatment with pemetrexed plus cisplatin for advanced nonsquamous non-small-cell lung cancer. Curr Med Res Opin 2018; 34: 865-871. 